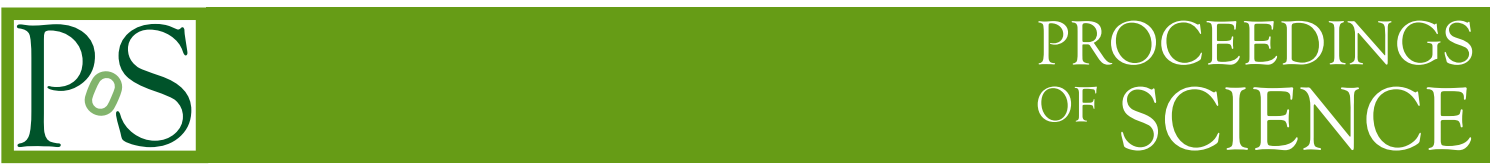

\title{
Dwingeloo Telescope has Fringes (Again)
}

\author{
E.P. Boven* \\ JIVE \\ E-mail: boven@jive.eu \\ C. van Tour, J.C.J. Koelemeij \\ VU/OPNT \\ R. Smets \\ SURFnet
}

A. Szomoru

JIVE

The Cleopatra work package of the ASTERICS project aims to address the common challenge of time and frequency distribution in distributed instruments and multi-messenger astronomy. We have made improvements to the open hardware 'White Rabbit' time and frequency distribution system to achieve better reach, stability and phase noise. Moreover, we are able to use White Rabbit over public fiber, co-existing with other traffic on different wavelengths. To demonstrate the achieved performance, we are conducting VLBI observations using the venerable Dwingeloo telescope, which will soon be connected to the hydrogen maser reference from the Westerbork Synthesis Radio Telescope, transported over $165 \mathrm{~km}$ of fiber in the SURFnet network. Using offthe-shelf software-defined-radio hardware, and the open-source GNU Radio program, we have recently achieved our first fringes between Westerbork, Jodrell Bank and Dwingeloo.

14th European VLBI Network Symposium \& Users Meeting (EVN 2018)

8-11 October 2018

Granada, Spain

*Also a CAMRAS volunteer 


\section{Time and Frequency Distribution using White Rabbit}

White Rabbit is a protocol for distributing time and frequency reference signals and control signals. It originated from CERN as an infrastructure for controlling the beam of the LHC. CERN have published the hardware and software components under an open license. The hardware is available from multiple vendors, and a large number of scientific projects have adopted White Rabbit (e.g. LHC, CTA, GSI, KM3NET, LHAASO, SKA and many more).

The original White Rabbit design offers sub-ns accuracy time distribution over distances of up to $10 \mathrm{~km}$. This can be extended up to approximately $100 \mathrm{~km}$ by using longer range pluggable optics. White Rabbit (WR) requires the use of bi-directional optical signal transport, where outward and return signals travel over the same fiber, and uses wavelength division multiplexing (WDM) to keep these signals separate. A WR link can be further extended by the use of bi-directional optical amplifiers[1]. Long haul optical telecommunication networks use all-optical amplifiers such as EDFAs to extend their reach. These amplifiers are guarded by isolators or circulators to ensure that any reflected light cannot re-enter the amplifier, which would cause it to start oscillating.

In our design, the WR optical signal is separated out from the other traffic on a fiber using a (coarse) WDM filter that encompasses both wavelengths in use, and separately amplified using its own bi-directional Silicon Optical Amplifiers (SOA). The amplified signal is then re-introduced in the outgoing fiber, and this is repeated at each amplifier location. A schematic diagram of the link that we established is shown in figure 1.

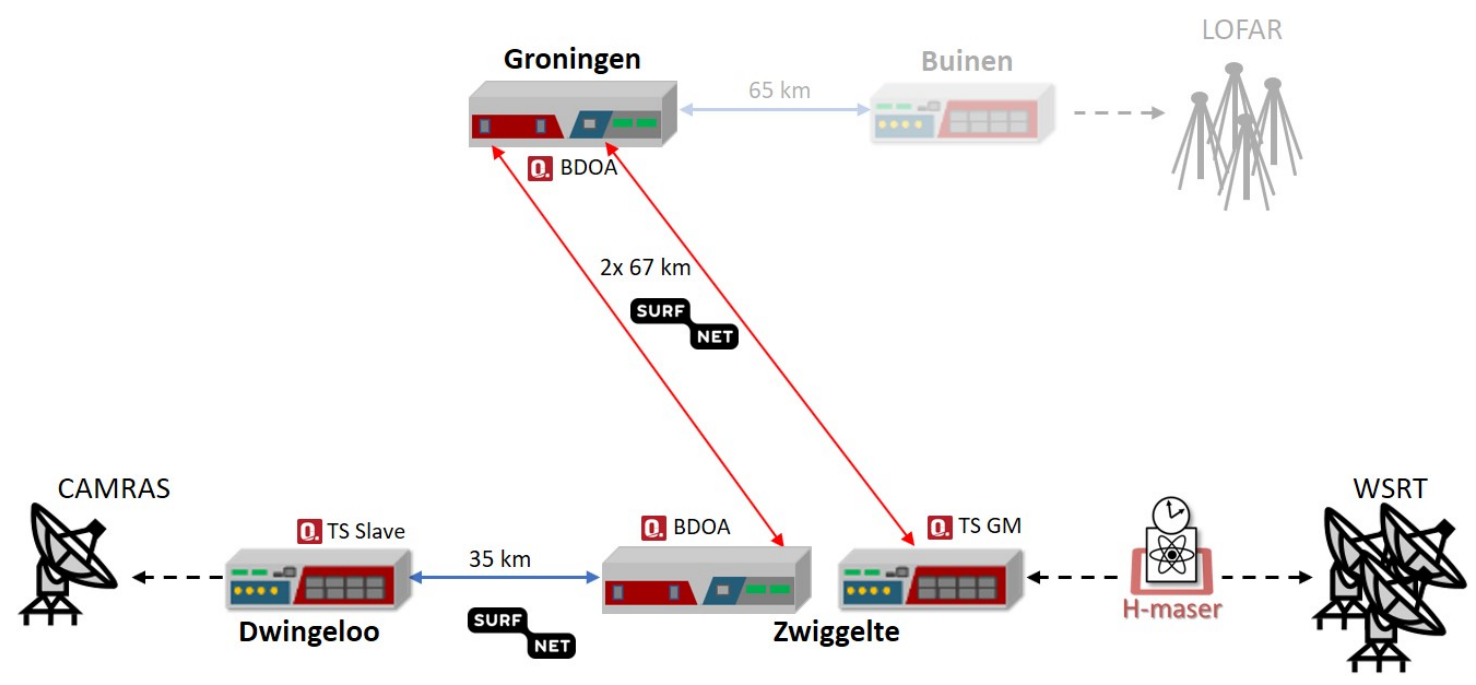

Figure 1: The link between WSRT and Dwingeloo, with the future extension to LOFAR. The entire link is situated in the northern region of the Netherlands.

The stability of the clock signal distributed by WR is not sufficient for VLBI. This is due to both the phase noise of the system, and the delay variations that arise due to wavelength variation in the laser, which through the dispersion of the fiber will cause uncompensated changes in propagation delay[2]. Three approaches were combined to improve the phase stability: Firstly, the use of temperature stabilised (dense WDM) lasers, which is a requirement for including the signal in 
the existing network, and limits the wavelength uncertainty to $0.1 \mathrm{~nm}$. Secondly, the WR switches were augmented with the 'Low Jitter Board' extension, also developed at CERN[3]. Finally the WR switch at the Dwingeloo telescope contains a clean-up oscillator to further reduce the short term phase noise of the link. The performance of the $134 \mathrm{~km}$ link WSRT-Groningen-WSRT is shown in figure 2, expressed as both an ADEV and MDEV measurement.
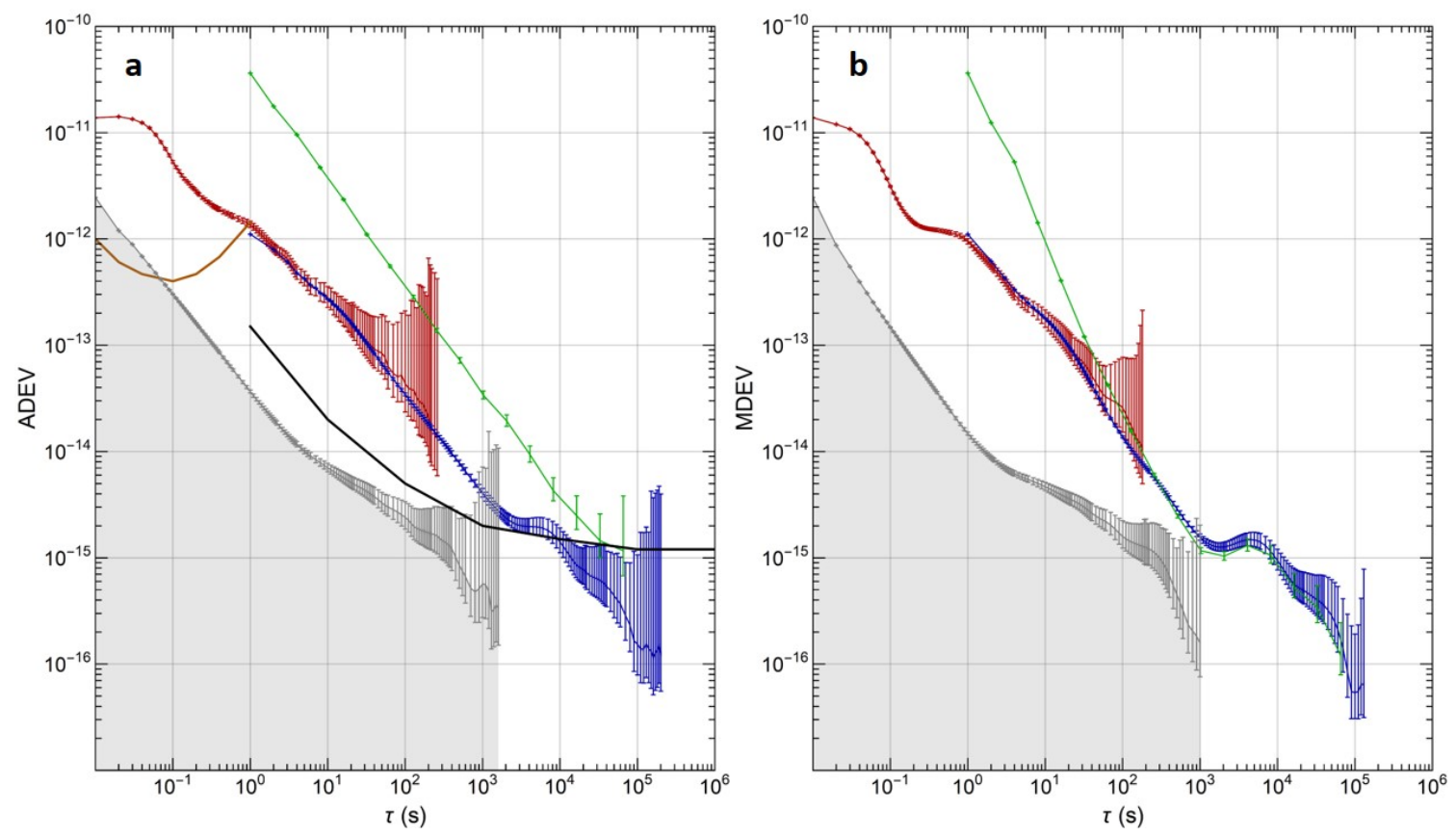

Figure 2: $\mathrm{ADEV}$ and MDEV on a $134 \mathrm{~km}$ link. Red: $50 \mathrm{~Hz}$ bandwidth, blue: $0.5 \mathrm{~Hz}$ bandwidth, green: TIC measurement on PPS, black: Maser performance (from datasheet), brown: performance of clean-up oscillator, grey: uncertainty for the frequency measurements (brown, blue, red).

\section{The Dwingeloo radio telescope}

The Dwingeloo radio telescope is a historic $25 \mathrm{~m}$ diameter radio telescope in the Netherlands. At its inauguration in 1956 it was the largest fully steerable radio telescope in the world. It had a very productive scientific career, and participated in the first European VLBI observations [4] in 1978, even before the EVN was formally constituted. But by the end of the 90's, the Dwingeloo telescope became disused as it was ecplised by the newer, larger Westerbork Synthesis Radio Telescope (WSRT).

It stood dormant for nearly a decade, until a group of volunteers adopted the instrument with permission of ASTRON, its owner. They founded the C.A. Muller Radio Astronomy Station (CAMRAS) society, and have worked hard on making it a fully working radio telescope again. With great support from ASTRON they have fully restored and rejuvenated the instrument. In 2011, the telescope was nominated a Dutch national monument which led to a complete overhaul of the mechanical structure. The receivers, signal processing, telescope control and anything else are now developed and maintained by the CAMRAS volunteers. They use it for outreach, education, pulsar and hydrogen observations, SETI, art projects and ham-radio (EME, Moon-bounce). 
Currently the telescope is equipped with a frontend for $400-440 \mathrm{MHz}(\mathrm{H}$ and V) and 1250 $1450 \mathrm{MHz}$ (LCP). Its close proximity to the JIVE and ASTRON offices makes it a great candidate for demonstrating time and frequency distribution for VLBI.

\section{A VLBI Backend in GNU Radio}

A complete VLBI backend would have been beyond the budget the ASTERICS project, let alone the CAMRAS foundation. The first author therefore created a VLBI receiver and formatter using an off-the-shelf Software Defined Radio (SDR), the Ettus X310 USRP, together with TwinRX receiver modules. The device in question is tunable from $10 \mathrm{MHz}$ to $6 \mathrm{GHz}$ and samples up to $80 \mathrm{MHz}$ of the spectrum at each of its 4 inputs. With $320 \mathrm{MHz}$ of sampled spectrum, or $160 \mathrm{MHz}$ per polarization, it should be possible to generate $1 \mathrm{~Gb} / \mathrm{s}$ of VLBI data (assuming the usual 2 bit sampling at the Nyquist rate).

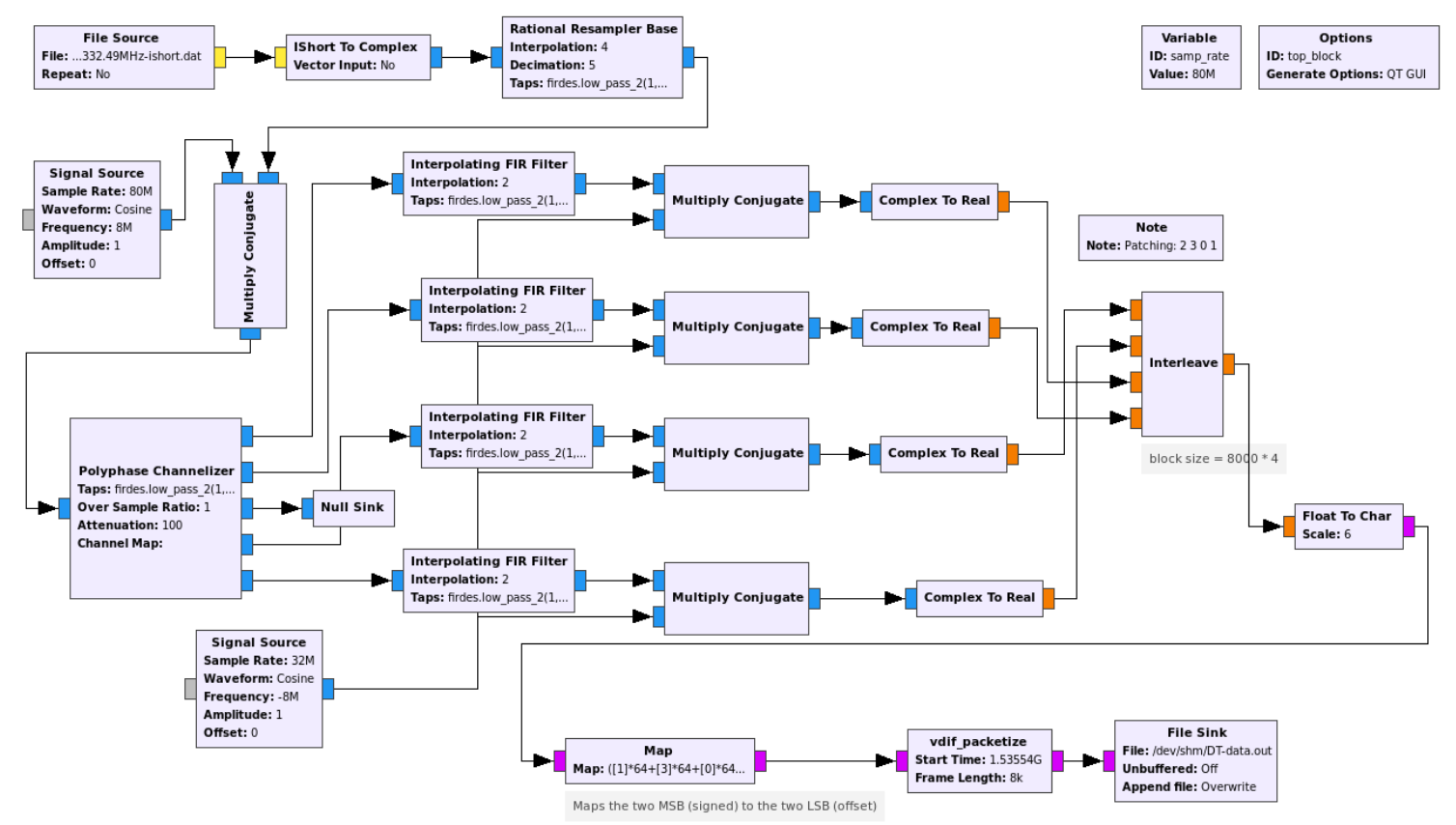

Figure 3: VLBI Formatter Flowchart for GNU Radio

The processing of the sampled data is carried out using the open source SDR framework 'GNU Radio' [5]. This software allows the rapid development of signal processing flows as either a Python based program, or a graphical flowchart. The flowchart design of the VLBI formatter is shown in Figure 3.

The processing starts on the top left, reading in the samples from a file and converting them into a stream of interleaved floating point numbers, representing complex samples. As the hardware provides $80 \mathrm{MHz}$ of bandwidth, oversampled at $100 \mathrm{MS} / \mathrm{s}$ (complex), the data is then re-sampled to $80 \mathrm{MS} / \mathrm{s}$. Eventually only $64 \mathrm{MHz}$ of the spectrum will be used, as 4 sub-bands containing $16 \mathrm{MHz}$ each. The "multiply conjugate" block, together with a sine generator to its left, is used to shift the whole spectrum by half a sub-band $(8 \mathrm{MHz})$, thereby putting both band-edges together in the 
same $16 \mathrm{MHz}$ band. The 'polyphase channelizer' block subsequently splits out the spectrum in 5 sub-bands of $16 \mathrm{MHz}$ each. The sub-band containing both band-edges is dropped by passing it into the 'null sink' block.

The four remaining sub-bands are each passed through a sequence of three blocks that convert the data from complex samples into real (USB) samples. This is done by doubling the sampling rate ('Interpolating FIR filter'), shifting the spectrum by $8 \mathrm{MHz}$ ('Multiply Conjugate') and then dropping the imaginary part of the spectrum in the 'Complex to Real' block. The sub-bands are now each sampled at $32 \mathrm{MS} / \mathrm{s}$, real samples, still representing $16 \mathrm{MHz}$ of spectrum each.

The 'Interleave' block takes the four input streams and outputs samples from each of the inputs in turn in blocks of 32,000 samples, which is the number of samples in one VDIF frame. 'Float to Char' converts the data to (signed) 8-bit data, and the 'Map' block implements a simple look-up table to go from signed bytes to the 2 bit offset binary encoding used in VDIF. The 'VDIF Packetize' block finally is a custom made block that takes the input samples, packs the 2 bit samples into bytes, and adds the VDIF header. To insert the VLBI timestamps, the block is provided with the start time of the recording as one of its parameters.

\section{4. 'Second' Fringes}
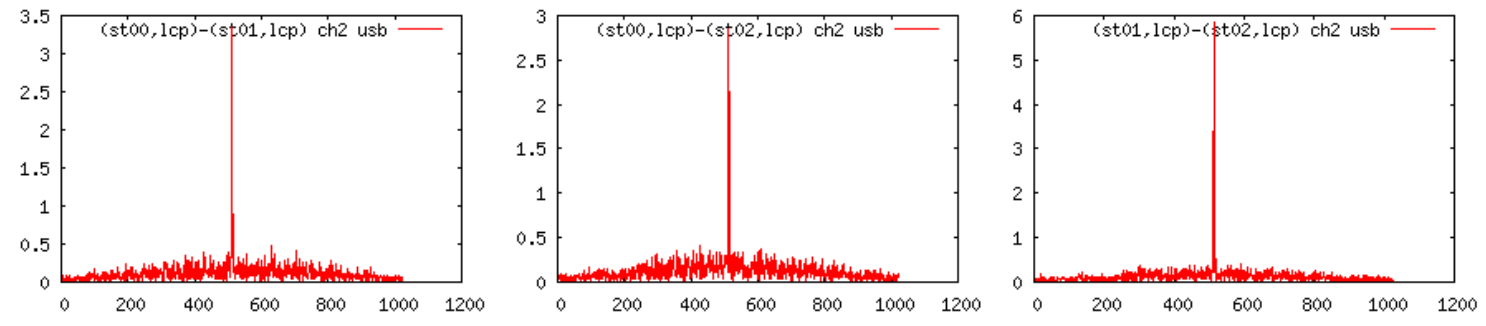

Figure 4: Fringes between Dwingeloo (st00), JBO Mk2 (st01) and WSRT (st02)

On August 25th 2018, 4C39.25 was observed by the Mark 2 telescope in Jodrell Bank, RT0 of the WSRT and the Dwingeloo telescope. The observing frequency range was $1300.49 \mathrm{MHz}-$ 1364.49 MHz, with Dwingeloo only observing in LCP (and supplying the same samples as RCP). As the ASTERICS connection for the hydrogen maser wasn't complete yet, we used a GPS locked Rubidium standard as a reference. The flowchart could not be run in real-time, so the observations for Dwingeloo consisted of recording $10 \mathrm{~s}$ (later $40 \mathrm{~s}$ ) of data into the main memory of a computer, and subsequently processing the data using GNU Radio. The resulting VDIF formatted data was sent to the fringetest server at JIVE, and processed using the standard workflow for FTP fringetests together with the data from the two other participating telescopes. Fringes were obtained as soon as we had valid data from all telescopes. These are depicted in Figure 4 and can also be seen on the EVN fringetest archive[6].

\section{Future Work}

With the first VLBI observation of the restored Dwingeloo telescope, we are close to completing the deliverables of the ASTERICS project and demonstrating that the reference clock for 
VLBI can be transported using an enhanced version of White Rabbit. The H-maser signal is already present in Dwingeloo, and only $300 \mathrm{~m}$ of digging remains to complete the fiber connection. Further work will include moving most of the VLBI formatter functionality into the FPGA that drives the SDR, using the RF Network-on-Chip (RFNOC)[7] extension to GNU Radio. This will allow VLBI and even e-VLBI at $1024 \mathrm{Mb} / \mathrm{s}$. Using the new VLBI capability in Dwingeloo, we will perform observations to research the suitability of the transported clock signal for VLBI.

\section{Acknowledgements}

ASTERICS is a project supported by the European Commission Framework Programme Horizon 2020 Research and Innovation action under grant agreement no. 653477.

\section{References}

[1] E.F. Dierikx, A.E. Wallin, T. Fordell, J. Myyry, P. Koponen, M. Merimaa, T.J. Pinkert, J.C.J. Koelemeij, H. Peek, R. Smets, "White Rabbit Precision Time Protocol on Long Distance Fiber Links", IEEE Transactions on Ultrasonics, Ferroelectrics and Frequency Control 63(7), 945-952 (2016).

[2] E.P. Boven, "DWDM Stabilized Optics for White Rabbit", Proceedings of the 2018 European Time and Frequency Forum. DOI: 10.1109/EFTF.2018.8409035

[3] Rizzi, M. e.a., "White Rabbit Clock Synchronization: Ultimate Limits on Close-In Phase Noise and Short-Term Stability Due to FPGA Implementation", EEE Transactions on Ultrasonics, Ferroelectrics, and Frequency Control, vol. 65, no. 9, pp. 1726-1737, Sept. 2018. DOI: 10.1109/TUFFC.2018.2851842

[4] Schilizzi, R.T. e.a., "High Resolution Observations of the Compact Central Component in the Giant Radio Source 3C236”, A\&A 77, 1-6 (1979)

[5] http://gnuradio.org/

[6] http://www.evlbi.org/tog/ftp_fringes/FT026/index.html

[7] https://kb.ettus.com/RFNoC 